\title{
Antitumor activity of the PD-1/PD-L1 binding inhibitor BMS-202 in the hu- manized MHC-double knockout NOG mouse
}

\author{
Tadashi Ashizawa ${ }^{1}$, Akira Izzuka ${ }^{1}$, Emiko Tanaka ${ }^{1}$, Ryota Kondou ${ }^{1}$, Haruo MiYata ${ }^{1}$, Chie Maeda ${ }^{1}$ Takashi \\ Sugino $^{2}$, Ken Yamaguchi ${ }^{3}$, Takayuki Ando ${ }^{4}$, Yoshinobu Ishikana ${ }^{5}$, Mamoru Ito ${ }^{6}$, and Yasuto AkiYama ${ }^{1}$ \\ ${ }^{1}$ Immunotherapy Division, Shizuoka Cancer Center Research Institute, ${ }^{2}$ Division of Pathology, ${ }^{3}$ Office of the president, Shizuoka Can- \\ cer Center Hospital, 1007 Shimonagakubo, Nagaizumi-cho, Sunto-gun, Shizuoka 411-8777, Japan; ${ }^{4}$ Shizuoka Institute of the Environ- \\ ment and Hygiene, 4-27-2 Kitaando, Aoi-ku, Shizuoka-shi, Shizuoka 420-8367, Japan; ${ }^{5}$ School of Pharmaceutical Sciences, University \\ of Shizuoka, 52-1 Yada, Suruga-ku, Shizuoka-shi, Shizuoka 422-8526, Japan; and ${ }^{6}$ Central Institute for Experimental Animals, \\ Kawasaki-ku, Kawasaki, Kanagawa 210-0821, Japan
}

(Received 25 September 2019; and accepted 15 October 2019)

\begin{abstract}
Recently, the first series of small molecule inhibitors of PD-1/PD-L1 were reported by BristolMyers Squibb (BMS), which were developed using a homogeneous time-resolved fluorescence (HTRF)-based screening investigation of the PD-1/PD-L1 interaction. Additional crystallographic and biophysical studies showed that these compounds inhibited the interaction of PD-1/PD-L1 by inducing the dimerization of PD-L1, in which each dimer binds one molecule of the stabilizer at its interface. However, the immunological mechanism of the antitumor effect of these compounds remains to be elucidated. In the present study, we focused on BMS-202 (a representative of the BMS compounds) and investigated its antitumor activity using in vitro and in vivo experiments. BMS-202 inhibited the proliferation of strongly PD-L1-positive SCC-3 cells $\left(\mathrm{IC}_{50} 15 \mu \mathrm{M}\right)$ and antiCD3 antibody-activated Jurkat cells $\left(\mathrm{IC}_{50} 10 \mu \mathrm{M}\right)$ in vitro. Additionally, BMS-202 had no regulatory effect on the PD-1 or PD-L1 expression level on the cell surface of these cells. In an in vivo study using humanized MHC-double knockout (dKO) NOG mice, BMS-202 showed a clear antitumor effect compared with the controls; however, a direct cytotoxic effect was revealed to be involved in the antitumor mechanism, as there was no lymphocyte accumulation in the tumor site. These results suggest that the antitumor effect of BMS-202 might be partly mediated by a direct off-target cytotoxic effect in addition to the immune response-based mechanism. Also, the humanized dKO NOG mouse model used in this study was shown to be a useful tool for the screening of small molecule inhibitors of PD-1/PD-L1 binding that can inhibit tumor growth via an immune-response-mediated mechanism.
\end{abstract}

Novel anticancer immunotherapies based on immune checkpoint blockade (ICB) have achieved remarkable success in the last several years. ICBbased cancer immunotherapy using monoclonal anti-

Address correspondence to: Dr Tadashi Ashizawa, Immunotherapy Division, Shizuoka Cancer Center Research Institute, 1007 Shimonagakubo, Nagaizumi-cho, Sunto-gun, Shizuoka 411-8777, Japan

Tel: +81-55-989-5222 (ext. 5330), Fax: +81-55-989-6085

E-mail: ta,ashizawa@scchr.jp bodies (mAbs) shows durable antitumor effects and produces long-lasting remission in patients with a variety of cancers $(7,12-14,17,21,25,26,28)$. However, $\mathrm{mAb}$ therapies, such as anti-programmed death (PD)-1/programmed death-ligand (PD-L)1 Abs, are expensive and still have a number of disadvantages, such as immunogenicity, which is typical of human mAbs, low oral bioavailability, poor solid-tumor tissue uptake, a limited half-life and, most importantly, mAb-related toxicity (immunerelated adverse effects, irAEs) $(10,33)$. In contrast, 
small-molecule compound-based therapeutics can have a greater affinity and specificity for targeted molecules than antibodies. Additionally, small molecule inhibitors have been shown to lack immunogenicity and to be good orally bioavailable and inexpensive. However, the development of small molecule inhibitors of the PD-1/PD-L1 pathway is currently far behind antibody development.

Recently, Zak et al. revealed the molecular features of the human PD-1/PD-L1 interaction based on the X-ray structure of the complex, and several hot spots located on the PD-L1 molecule were shown to be involved in the formation of the complex (15, $30)$.

To date, several small molecules, macrocyclic peptides and peptide mimetics targeting the PD-1/PD-L1 interaction have been reported $(8,18,19)$, primarily in patent applications, but almost no fully validated and qualified therapeutics exist. Recently, the binding action and biological activities of potent small molecule inhibitors of PD-1/PD-L1 have been reported by Bristol-Myers Squibb (BMS) (1, 31, 32). The first series of small molecule inhibitors of PD-1/PD-L1 interaction identified using a homogeneous time-resolved fluorescence (HTRF) binding assay-based screening method was reported in a patent document (31). In the crystallographic study, BMS-202 was shown to inhibit the interaction by inducing the dimerization of PD-L1. Gunik et al. demonstrated that BMS-202 is located at the center of the PD-L1 homodimer in a deep hydrophobic pocket between the two-PD-L1 molecules (11).

In the present study, we focused on BMS-202 (a representative BMS compound with biological activity) and investigated its antitumor activity using in vitro and in vivo experiments. In particular, for the in vivo studies, we used humanized major histocompatibility complex (MHC)-double knockout (dKO) NOD/Shi-scid-IL2r $\gamma^{\text {null }}$ (NOG) mice (29), and investigated the immunological effect of the PD-1/PD-L1 inhibitor BMS-202 on human lymphoma SCC-3 cells.

\section{MATERIALS AND METHODS}

Chemicals. The BMS-202 (Fig. 1A) compounds were purchased from Selleck Chemicals (Houston, TX, USA) and Ark Pharm, Inc. (Arlington Heights, IL, USA). The former was used for the in vitro (cell proliferation assay and flow cytometry) experiment. The latter was used for the cell proliferation assay and in vivo experiments. The biological activities of both compounds were compared in the cell prolifer- ation assay. These compounds were suspended in a sterile $0.5 \%$ methyl cellulose $400 \mathrm{cp}$ solution (Wako, Tokyo, Japan) or dissolved in dimethyl sulfoxide (DMSO) (Wako).

Cell lines and reagents. The human lymphoma SCC-3 and Jurkat cell lines were purchased from JCRB (Saito, Osaka, Japan) and ATCC (Manasas, VA, USA), respectively, and maintained in RPMI 1640 (SIGMA, St. Louis, MO, USA) supplemented with $10 \%$ fetal bovine serum (FBS; Thermo Fisher Scientific, Waltham, MA, USA). Anti-programmed death-ligand 1 (PD-L1, CD274)-APC and anti-PD-1 (CD279)-APC antibodies purchased from BioLegend Inc. (San Diego, CA, USA) were used for flow cytometric analysis. The anti-CD3 antibody was purified from the supernatant of the anti-human CD3 (OKT3) hybridoma cell line (ATCC) and used for the stimulation of Jurkat cells.

Cell proliferation assay. Cell proliferation was examined using the WST-1 assay (Dojin Kagaku Corp., Kumamoto, Japan) as described previously (2). Briefly, $1 \times 10^{4}$ SCC-3 cells were seeded in a 96well microculture plate (Corning Inc., Corning, NY), and compounds at concentrations ranging from 0.25 to $100 \mu \mathrm{M}$ were added. A total of $1 \times 10^{4}$ Jurkat cells were seeded in a 96-well microculture plate coated with anti-human CD3 antibody $(2 \mu \mathrm{g} / \mathrm{mL})$ at $4^{\circ} \mathrm{C}$ overnight and incubated with the BMS-202 compound. After 4 days, the WST-1 substrate was added to the culture, and the optical density (OD) was measured at 450 and $620 \mathrm{~nm}$ using an immunoreader (Nivo, PerkinElmer Inc., Waltham, MA, USA).

Flow cytometry analysis. SCC-3 or Jurkat cells treated with $25 \mu \mathrm{M}$ BMS-202 compound for $24 \mathrm{~h}$ were washed and stained with anti-PD-1 or antiPD-L1 antibody for $15 \mathrm{~min}$ at $4^{\circ} \mathrm{C}$. The staining method was described previously (3). The stained cells were analyzed on a FACS Canto II flow cytometer (BD Biosciences, San Diego, CA, USA).

Development of humanized NOG-dKO mice. Sixweek-old NOG-dKO mice were kindly supplied by Dr. Mamoru Ito, the Central Institute for Experimental Animals (Kawasaki, Japan). All animals were cared for and treated humanely according to the Guidelines for the Welfare and Use of Animals in Cancer Research, and the experimental procedures were approved by the Animal Care and Use Committee of Shizuoka Cancer Center Research In- 
A<smiles>COc1nc(CNCCNC(C)=O)ccc1COc1cccc(-c2ccccc2)c1C</smiles>

B
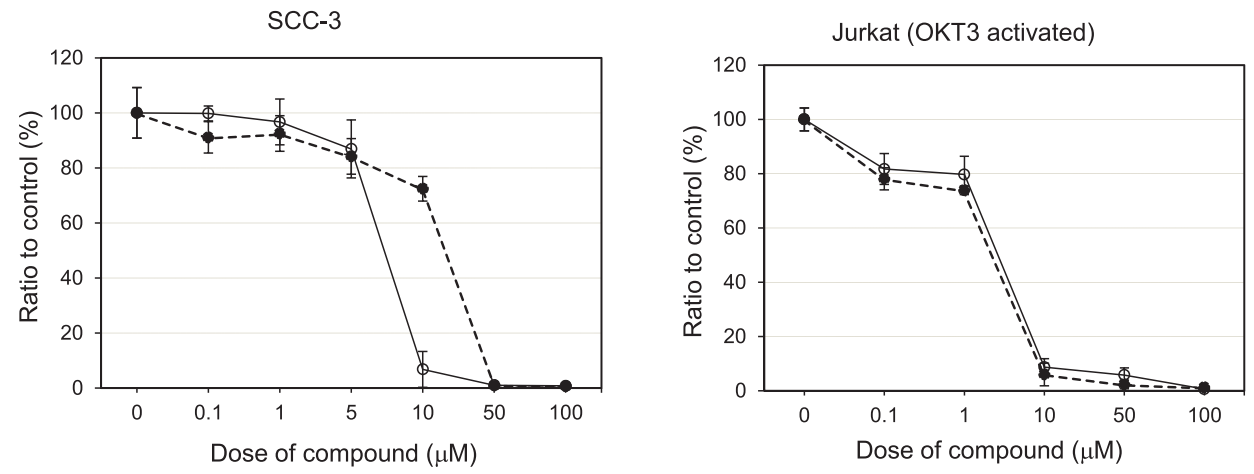

Fig. 1 Chemical structure of BMS-202 and effect of BMS-202 on cell proliferation. (A) Formula, $\mathrm{C}_{25} \mathrm{H}_{29} \mathrm{~N}_{3} \mathrm{O}_{3}$. Molecular weight: 419.52. (B) The proliferation of SCC-3 and anti-CD3 antibody-stimulated Jurkat cells was inhibited by BMS-202. Each point represents the mean \pm SD of triplicates. Open circle; BMS-202 (Selleck), closed circle; BMS-202 (Ark Pharm).

stitute.

The experimental procedures used were described previously (3). Briefly, eight-week-old NOG-dKO mice were irradiated with X-rays (2.5 Gy) and $1 \times 10^{7}$ human peripheral blood mononuclear cells (PBMCs) with the human leukocyte antigen (HLA)-A 0201 genotype were intravenously (i.v.) administered to each mouse on day 0 . A total of $2 \times 10^{5}$ SCC-3 cells with the HLA-A 0201 genotype were subcutaneously transplanted into three mice in each group on day 1. The daily injection of BMS-202 at a dosage of $20 \mathrm{mg} / \mathrm{kg}$ was started on day 14 and administered a total of 9 times. The tumor volume was calculated based on the National Cancer Institute formula as follows: tumor volume $\left(\mathrm{mm}^{3}\right)=$ length $(\mathrm{mm}) \times[$ width $(\mathrm{mm})]^{2} \times 1 / 2$.

Immunohistochemistry (IHC). Anti-CD4 and anti-CD8 antibodies (Thermo Fisher Scientific), anti-granzyme $\mathrm{B}$ antibody (DAKO), anti-CD204 antibody (TransGenic Inc.), and anti-PD-L1 antibody (BioLegend Inc) were purchased and used for IHC analysis as described previously (3).

Statistical analysis. Significant differences were analyzed using Student's $t$-test. Values of $P<0.05$ were considered to be indicative of statistical significance.

\section{RESULTS}

BMS-202 inhibited cell proliferation of SCC-3 and Jurkat cells

The $\mathrm{IC}_{50}$ value for the cytotoxic activity of the PD-1/ PD-L1 binding inhibitor BMS-202 in SCC-3 cells and anti-CD3 Ab-activated Jurkat cells was $15 \mu \mathrm{M}$ and $10 \mu \mathrm{M}$, respectively (Fig. 1B). BMS-202 showed moderate cytotoxicity similar to other BMS-series small compounds of 2-methyl-3-biphenyl-methanol moiety-containing compounds (BMS-8, -37 and -242), had $\mathrm{EC}_{50}$ values between 3 and $10 \mu \mathrm{M}$ (22).

BMS-202 had no effect on PD-1 or PD-L1 expression Flow cytometry analysis of BMS-202-treated SCC-3 cells or activated Jurkat cells demonstrated that BMS-202 had no regulatory effects on the PD-1 or PD-L1 expression level at the cell surface (Fig. 2).

BMS-202 inhibited the tumor growth of SCC-3 cells in humanized NOG-dKO mice

The experimental design and treatment schedule used for BMS-202 therapy are shown in Fig. 3A. BMS-202 showed $41 \%$ growth inhibitory activity against humanized mouse-transplanted human lymphoma SCC-3 cells (Fig. 3B). In addition, the body weight-reducing effect, which was a measure of the 
PD-1
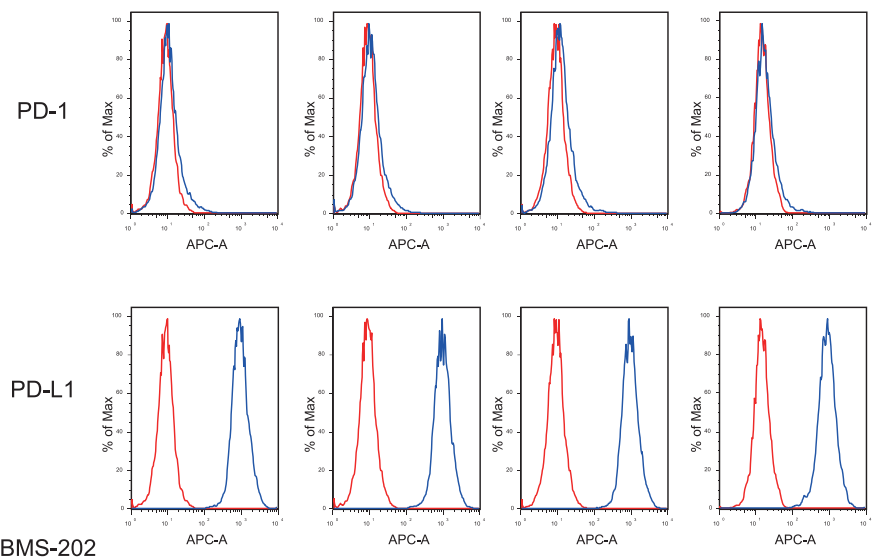

$(\mu \mathrm{M})$

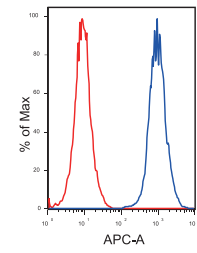

0.1

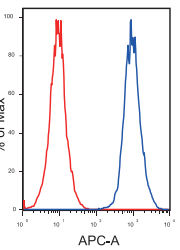

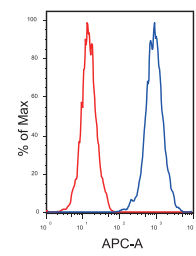

10
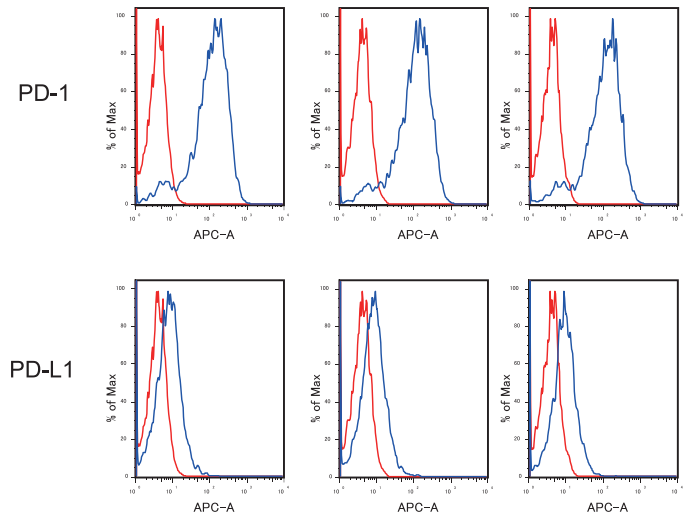

BMS-202

$(\mu \mathrm{M})$

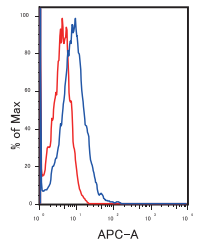

0.1

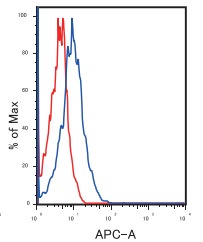

1

Fig. 2 PD-1/PD-L1 expression levels on the cell surface. BMS-202 showed no significant effect on the PD-1/PD-L1 expression level. The PD-1 and PD-L1 expression levels measured by flow cytometry on SCC-3 and anti-CD3 antibody-stimulated Jurkat cells treated with various doses of BMS-202, are shown. Red line: isotype control, blue line: anti-PD-1 or anti-PD-L1 antibody.

A

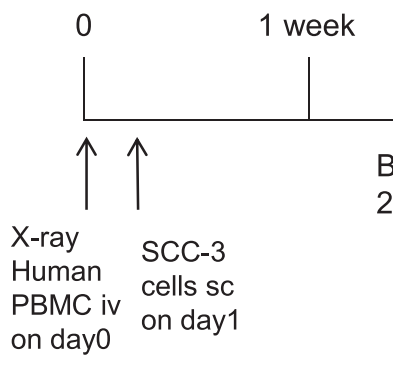

2 weeks $\quad 3$ weeks $\quad 4$ weeks

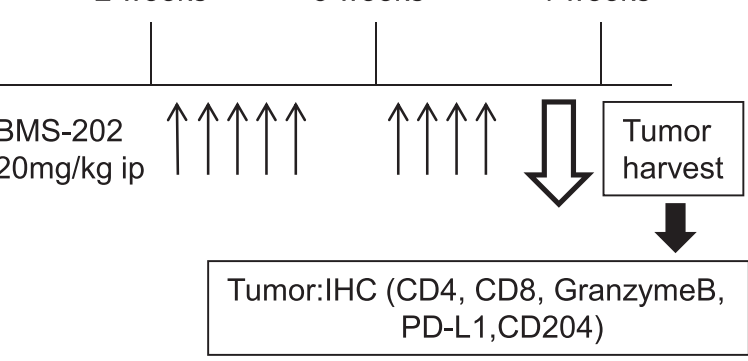

B

C
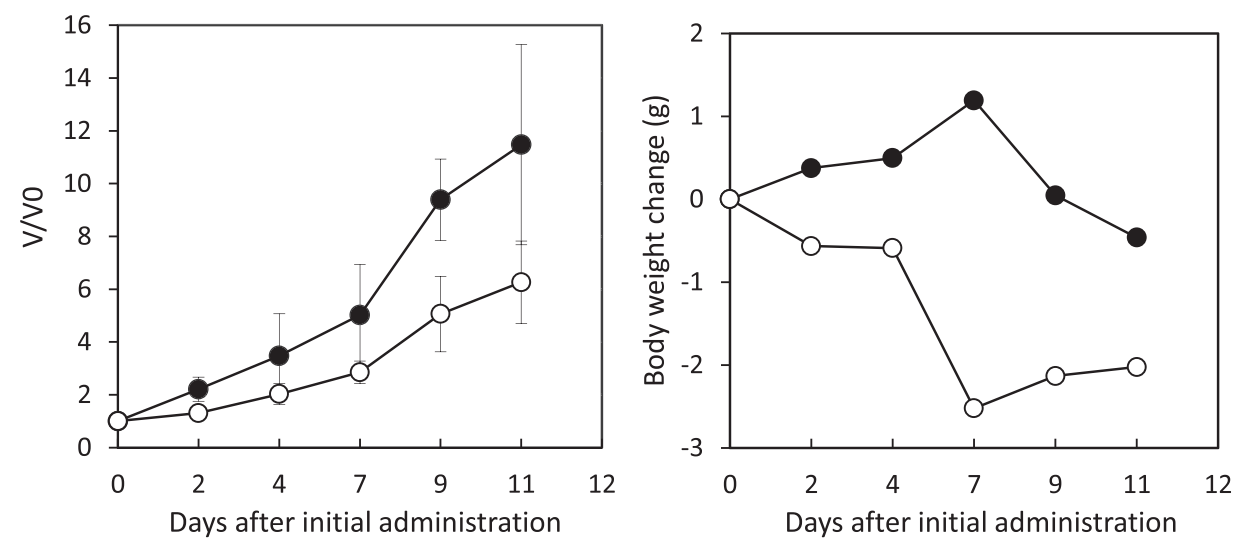

Fig. 3 Inhibitory effect of BMS-202 on the growth of SCC-3 tumors in vivo. (A) Experimental design and treatment schedule used for BMS-202 therapy of SCC-3 tumors in humanized MHC-dKO NOG mice. Beginning on day 14, BMS202 was administered intraperitoneally for 9 days. (B) VIVO values of BMS-202-treated SCC-3 tumors $(n=3)$ are shown. The efficacy of BMS-202 treatment is expressed as the mean V/VO value, where $V$ is the tumor volume on the day of evaluation and $V O$ is the tumor volume on the day of treatment. (C) Body weight change in BMS-202-treated mice bearing SCC-3 tumors. Closed circle; control, open circle; BMS-202-treated group. Each point represents the mean \pm SD value derived from the measurement of SCC-3 tumors in three mice. 

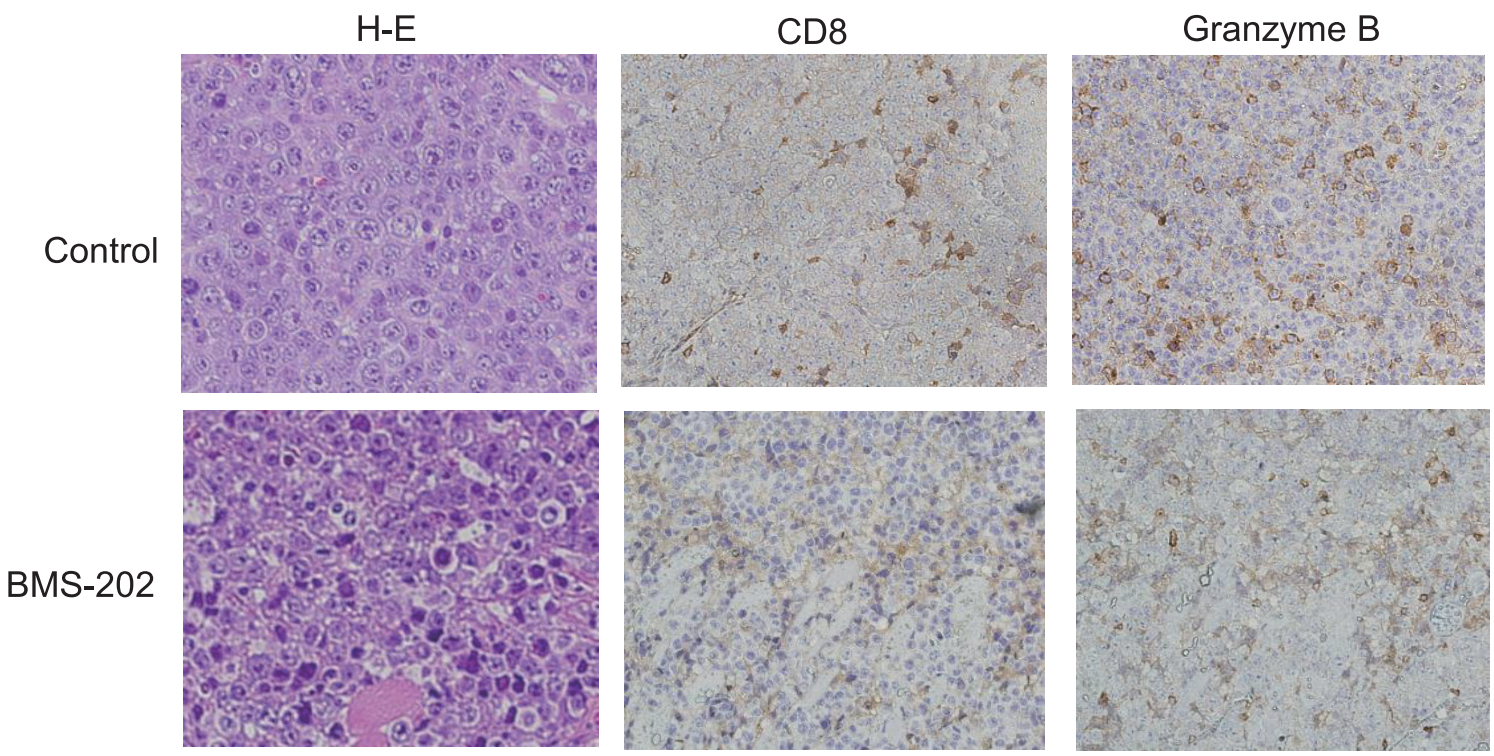

\section{CD204}
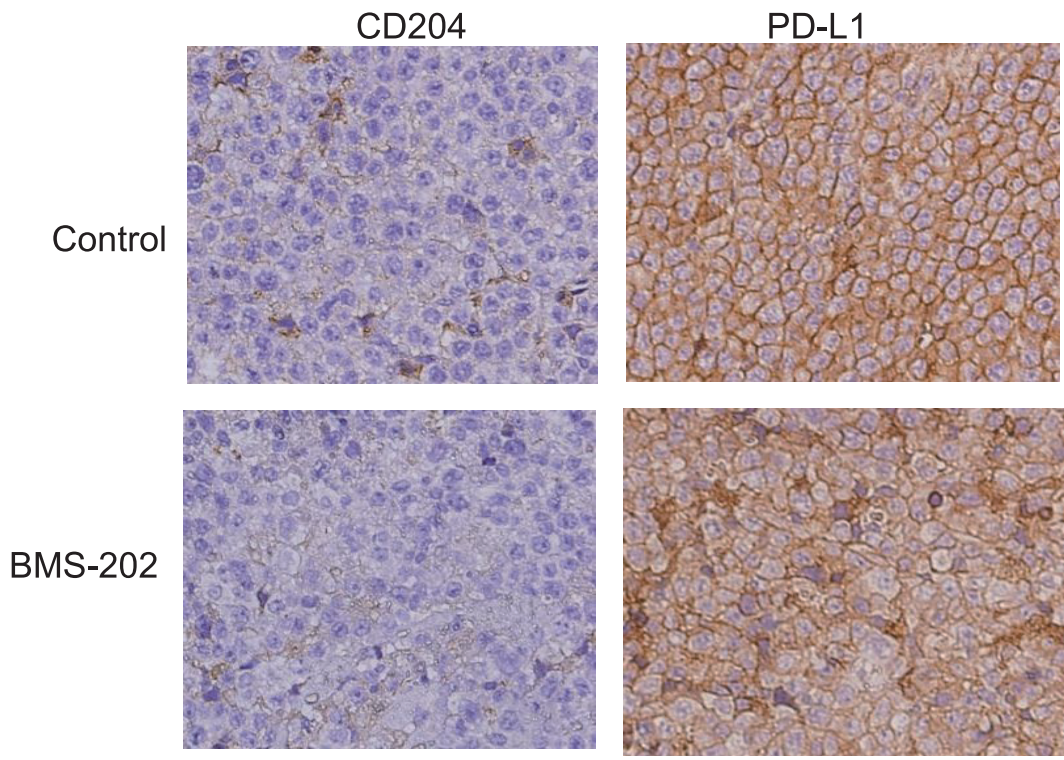

Fig. 4 Effect of BMS-202 on SCC-3 tumors and infiltrating immune cells in SCC-3 tumors. Images of control and BMS202-treated tumors stained with H\&E and anti-CD8, anti-granzyme B, CD204 and anti-PD-L1 antibodies. Magnification: $\times 200$.

systemic toxicity, was stronger in the BMS-202-administered group than in the control group (Fig. 3C)

BMS-202 did not promote TIL accumulation in tumors

SCC-3 tumors treated with BMS-202 demonstrated increased necrosis and poor viability (Fig. 4) compared with the control group. Generally, the induction of CTL (cytotoxic T lymphocyte) activity killing tumor cells and promotion of intratumoral infiltration of immune effector cells like CTLs are immune responses frequently observed in patients with can- cer treated with antibodies targeting immune checkpoint molecule (27). However, in the present study, the numbers of tumor-infiltrating lymphocytes (TILs), which consisted of $\mathrm{CD}^{+}$and granzyme $\mathrm{B}^{+} \mathrm{T}$ cells in the central area of the tumor, was markedly diminished compared to that in the control (Fig. 5). These results might suggest that a direct cytotoxic effect rather than immune response was likely to be involved in the antitumor mechanisms. Furthermore, PD-L1 protein staining was reduced by damage to the tumor tissue. Tumor-associated macrophages (TAM), which infiltrate into tumor tissues, are well 

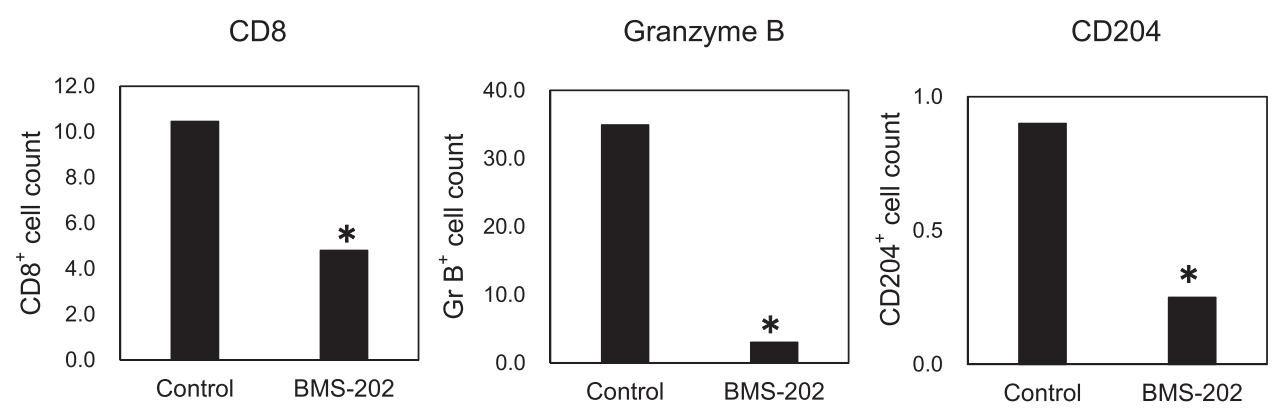

Fig. 5 Effect of BMS-202 on the number of infiltrating immune cells in SCC-3-tumors. More than 10 areas of tumor in each tumor section stained with various antibodies were analyzed at high magnification $(\times 200)$ using Winroof image analysis software. The positive cell counts per field were compared between the control and BMS-202-treated groups. Each column shows the mean \pm SD value. ${ }^{*} P<0.05$, statistically significant.

known to be involved in cancer progression. CD204 is a representative pro-tumor marker for M2-type TAMs (24). There was a tendency towards a decrease in the CD204-positive cell number in BMS-202treated tumors compared to control tumors (Fig. 5).

\section{DISCUSSION}

Recently, several small molecule inhibitors targeting the PD-1/PD-L1 pathway that are based on peptidomimetics $(9,23)$ and macrocyclic peptides $(16)$ have been reported. The small molecule inhibitors developed by BMS are structural derivatives of (2-methyl3-biphenylyl)methanol. The capability to block PD-1/ PD-L1 complex formation was evaluated using the HTRF assay. Among the most potent inhibitors, BMS-202 inhibited the formation of the PD-1/PD$\mathrm{L} 1$ complex, with an $\mathrm{IC}_{50}$ value of $0.018 \mu \mathrm{M}$ (31). Despite the fact that BMS-202 has poor druglike properties and is unlikely to become a lead compound, the results show that targeting the PD-1/PDL1 interaction surface is feasible not only by using anti-PD-1/PD-L1 antibodies, but also by using anti-PD-1/PD-L1 small molecule compounds (11). However, a substantial evidence that BMS-202 can regulate tumor growth based on specific immunological mechanisms has not been proven at the preclinical level, including animal models.

Our study shows the in vitro and in vivo activity of the small molecule PD-1/PD-L1 inhibitor BMS202 in preclinical studies. To define the highest concentrations of BMS-202 that could be used in vitro assays, the cytotoxicity of BMS-202 was evaluated by a proliferation assay using strongly PD-L1-positive SCC-3 cells and WST-1. Compared with the most cytotoxic derivatives of (2-methyl-3-biphenylyl)methanol, such as BMS-37 and BMS-242, BMS202 showed moderate cytotoxicity, with an $\mathrm{IC}_{50}$ value of $13.9 \mu \mathrm{M}$.

Two optimized (2,3-dihydro-1,4-benzodioxinebased inhibitors, BMS-1001 and BMS-1166, presented significantly improved cytotoxic properties (22), unlike the other three compounds tested (BMS-202, BMS-37 and BMS-242). Additionally, BMS-1001 and BMS-1166 restored the activation of effector Jurkat T cells; however, their potency was significantly weaker than that shown by anti-PD-1 and antiPD-L1 antibodies (22). Further optimization based on the structural data may lead to the development of more potent PD-1/PD-L1 inhibitors. However, with regard to these compounds, no biological experiments indicating the immunological activity have been published to date.

Interestingly, a novel small molecule dual inhibitor of immune checkpoint PD-L1 and V-domain Ig-containing suppressor of T-cell activation (VISTA), CA-170, was recently investigated in a phase 1 trial of advanced solid tumors and lymphomas $(6,10)$.

In the present study, we used humanized NOGdKO mice transplanted with human PBMCs and SCC-3 human lymphoma cells and found that BMS202 treatment efficiently inhibited the growth of transplanted tumors. Moreover, the promotion by BMS-202 of tumor-infiltrating lymphocytes (TIL) accumulation within the tumor was not observed; rather, TIL numbers were diminished in BMS-202treated tumors compared with control tumors as shown in Fig. 4. These results suggest that the antitumor effect of BMS-202 might be partly mediated by a direct off-target cytotoxic effect in addition to the immune response-based mechanism. Previously, we demonstrated in humanized NOG-dKO mice that anti-PD-1 antibody inhibited the growth of SCC-3 tumors through the promotion of active effector $\mathrm{T}$ cell accumulation in the tumor and reduced weight loss induced by SCC-3 tumor progression (4). In 
contrast, BMS-202 administration resulted in remarkable TIL depletion and substantial weight loss in the current study.

Recently, BMS-202 has been reported to show a potent PD-1/PD-L1 protein binding inhibition, but result in weak cell-based PD-1/PD-L1 blockade activity and strong cytotoxicity activity (5), which might support our observation in vivo study using humanized dKO NOG mouse. Therefore, based on these observations, we are not aiming at developing BMS202 as a PD-1/PD-L1 binding inhibitor.

The humanized NOG-dKO mouse model has clear advantages over other immune-deficient mouse models that use patient-derived immune cells and tumor cells. In in vivo study investigating the effects of a STAT3 inhibitor (STX-0119) and anti-PD-1 antibody on human immune cells $(3,4)$, the humanized NOG-dKO model was more successful compared to a conventional nude mice model. This demonstrated that this model will be a good tool for screening small molecule inhibitors of PD-1/PD-L1 binding and possibly lead to the development of potent novel immune-therapeutics for malignancies.

\section{Acknowledgements}

The authors thank Dr. Kouji Maruyama and staff at the animal facility of Shizuoka Cancer Center for technical assistance.

\section{Funding}

This work was supported by a grant to Tadashi Ashizawa from JSPS KAKENHI (Grant Number 18K07315), Japan.

Availability of data and materials

All data generated or analyzed during this study are included in this published article.

Ethics approval and consent to participate

All animals were cared for and treated humanely according to the Guidelines for the Welfare and Use of Animals in Cancer Research, and the experimental procedures were approved by the Animal Care and Use Committee of Shizuoka Cancer Center Research Institute. The clinical experiments using peripheral blood mononuclear cells (PBMCs) derived from glioma patients were approved by the Institutional Review Board of Shizuoka Cancer Center (Authorization Number: 24-29).

\section{COMPETING INTERESTS}

The authors declare that they have no competing interests.

\section{REFERENCES}

1. Abdel-Magid AF (2015) Inhibitors of the PD-1/PD-L1 pathway can mobilize the immune system: an innovative potential therapy for cancer and chronic infections. ACS Med Chem Lett 6, 489-490.

2. Akiyama Y, Nonomura C, Kondou R, Miyata H, Ashizawa T, Maeda C, Mitsuya K, Hayashi N, Nakasu Y and Yamaguchi $\mathrm{K}$ (2016) Immunological effects of the anti-programmed death-1 antibody on human peripheral blood mononuclear cells. Int J Oncol 49, 1099-1107.

3. Akiyama $\mathrm{Y}$, Nonomura $\mathrm{C}$, Ashizawa $\mathrm{T}$, Iizuka A, Kondou R, Miyata H, Sugino T, Mitsuya K, Hayashi N, Nakasu Y, et al. (2017) The anti-tumor activity of the STAT3 inhibitor STX0119 occurs via promotion of tumor-infiltrating lymphocyte accumulation in temozolomide-resistant glioblastoma cell line. Immunol Lett 190, 20-25.

4. Ashizawa T, Iizuka A, Nonomura C, Kondou R, Maeda C, Miyata $\mathrm{H}$, Sugino $\mathrm{T}$, Mitsuya $\mathrm{K}$, Hayashi $\mathrm{N}$, Nakasu et al. (2017) Antitumor effect of programmed death-1 (PD-1) blockade in humanized the NOG-MHC double knockout mouse. Clin Cancer Res 23, 149-158.

5. Basu S, Yang J, Xu B, Magiera-Mularz K, Skalniak L, Musielak B, Kholodovych V, Holak TA and Hu L (2019) Design, synthesis, evaluation, and structural studies of C2-symmetric small molecule inhibitors of programmed cell death-1/ programmed death-ligand 1 protein-protein interaction. $J$ Med Chem 62, 7250-7263.

6. Bojadzic D and Buchwald P (2018) Toward small-molecule inhibition of protein-protein interactions: general aspects and recent progress in targeting costimulatory and coinhibitory (immune checkpoint) interactions. Curr Top Med Chem 18, 674-699.

7. Brahmer JR, Tykodi SS, Chow LQ, Hwu WJ, Topalian SL, Hwu P, Drake CG, Camacho LH, Hauh J, Odunsi K, et al. (2012) Safety and activity of anti-PD-L1 antibody in patients with advanced cancer. $N$ Engl J Med 366, 2455-2465.

8. Chang HN, Liu BY, Qi YK, Zhou Y, Chen YP, Pan KM, Li WW, Zhou XM, Ma WW, Fu CY, et al. (2015) Blocking of the PD-1/PD-L1 interaction by a D-peptide antagonist for cancer immunotherapy. Angew Chem Int Ed Engl 54, 1176011764.

9. Chen T, Li Q, Liu Z, Chen Y, Feng F and Sun H (2019) Peptide-based and small synthetic molecule inhibitors on PD-1/ PD-L1 pathway: a new choice for immunotherapy? Eur $J$ Med Chem 161, 378-398.

10. Geng Q, Jiao P, Jin P, Su G, Dong J and Yan B (2018) PD-1/ PD-L1 inhibitors for immune-oncology: From antibodies to small molecules. Curr Pharm Des 23, 6033-6041.

11. Guzik K, Zak KM, Grudnik P, Magiera K, Musielak B, Torner R, Skalniak L, Domling A, Dubin G and Holak TA (2017) Small-molecule inhibitors of the programmed cell death-1/programmed death-ligand 1 (PD-1/PD-L1) interaction via transiently induced protein states and dimerization of PDL1. J Med Chem 60, 5857-5867.

12. Hodi FS, O'Day SJ, McDermott DF, Weber RW, Sosman JA, Haanen JB, Gonzalez R, Robert C, Schadendorf D, Hassel JC, et al. (2010) Improved survival with ipilimumab in pa- 
tients with metastatic melanoma. $N$ Engl $J$ Med 363, 711723.

13. Hoos A (2016) Development of immune-oncology drugs from CTLA4 to PD1 to the next generations. Nat Rev Drug Discov 15, 235-257.

14. Khalil DN, Smith EL, Brentjens RJ and Wolchok JD (2016) The future of cancer treatment: immunomodulation, CARs and combination immunotherapy. Nat Rev Clin Oncol 13, 273-290.

15. Lin DY, Tanaka Y, Iwasaki M, Gittis AG, Su HP, Mikami B, Okazaki T, Honjo T, Minato N and Garboczi DN (2008) The PD-1/PD-L1 complex resembles the antigen-binding Fv domains of antibodies and T cell receptors. Proc Natl Acad Sci USA 105, 3011-3016.

16. Magiera-Mularz K, Skalniak L, Zak KM, Musielak B, Rudzinska-Szostak E, Berlicki L, Kocik J, Grudnik P, Sala D, Zarganes-Tzitzikas T, et al. (2017) Bioactive macrocyclic inhibitors of the PD-1/PD-L1 immune checkpoint. Angew Chem Int Ed Engl 56, 13732-13735.

17. Mahoney KM, Rennert PD and Freeman GJ (2015) Combination cancer immunotherapy and new immunomodulatory targets. Nat Rev Drug Discov 14, 561-584.

18. Patil SP, Yoon SC, Aradhya AG, Hofer J, Fink MA, Enley ES, Fisher JE, Herb MC, Kingos A, Proulx JT, et al. (2018) Macrocyclic compounds from ansamycin antibiotic class as inhibitors of PD-1PD-L1 protein-protein interaction. Chem Pharm Bull 66, 773-778.

19. Sakikumar PG and Ramachandra M (2018) Small-molecule immune checkpoint inhibitors targeting PD-1/PD-L1 and other emerging checkpoint pathways. BioDrugs 32, 481-497.

20. Shaabani S, Huizinga HPS, Butera R, Kouchi A, Guzik K, Magiera-Mularz K, Holak TA and Dömling A (2018) A patent review on PD-1/PD-L1 antagonists: small molecules, peptides, and macrocycles (2015-2018). Expert Opin Ther Pat 28, 665-678.

21. Sharma P and Allison JP (2015) The future of immune checkpoint therapy. Science 348, 56-61.

22. Skalniak L, Zak KM, Guzik K, Mariera K, Musielak B, Pachota M, Szelazek B, Kocik J, Grudnik P, Tomala M, et al. (2017) Small-molecule inhibitors of PD-1/PD-L1 immune checkpoint alleviate the PD-L1-induced exhaustion of T-cells. Oncotarget 8, 72167-72181.

23. Sasikumar PG, Ramachandra RK, Adurthi S, Dhudashia AA, Vadlamani S, Vemula K, Vunnum S, Satyam LK, Samiulla DS, Subbarao K, et al. (2019) A rationally designed peptide antagonist of the PD-1 signaling pathway as an immunomod- ulatory agent for cancer therapy. Mol Cancer Ther, doi: 10.1158/1535-7163.MCT-18-0737.

24. Shigeoka M, Urakawa N, Nakamura T, Nishio M, Watajima T, Kuroda D, Komori T, Kakeji Y, Semba S and Yokozaki H (2013) Tumor associated macrophage expressing CD204 is associated with tumor aggressiveness of esophageal squamous cell carcinoma. Cancer Sci 104, 1112-1119.

25. Topalian SL, Hodi FS, Brahmer JR, Gettinger SN, Smith DC, McDermott DF, Powderly JD, Carvajal RD, Sosman JA, Atkins MB, et al. (2012) Safety, activity, and immune correlates of anti-PD-1 antibody in cancer. $N$ Engl J Med 366, $2443-2454$

26. Topalian SL, Drake CG and Pardoll DM (2015) Immune checkpoint blockade: a common denominator approach to cancer therapy. Cancer Cell 27, 450-461.

27. Taube JM, Klein A, Brahmer JR, Xu H, Pan X, Kim JH, Chen L, Pardoll DM, Topalian SL and Anders RA (2014) Association of PD-1, PD-1 ligands, and other features of the tumor immune microenvironment with response to anti-PD-1 therapy. Clin Cancer Res 20, 5064-5074.

28. Wolchok JD, Kluger H, Callahan MK, Postow MA, Rizvi NA, Lesokhin AM, Segal NH, Ariyan CE, Gordon RA, Reed $\mathrm{K}$, et al. (2013) Nivolomab plus ipilimumab in advanced melanoma. N Engl J Med 369, 122-133.

29. Yaguchi T, Kobayashi A, Inozume T, Morii K, Nahumo H, Nishio H, Iwata T, Ka Y, Katano I, Ito R, et al. (2018) Human PBMC-transferred murine MHC class I/II-deficient NOG mice enable long-term evaluation of human immune responses. Cell Mol Immunol 15, 953-962.

30. Zak KM, Grudnik P, Mariera K, Dömling A, Dubin G and Holak TA (2017) Structural biology of the immune checkpoint receptor PD-1 and its ligand PD-L1/PD-L2. Structure 25, 1163-1174.

31. Zak KM, Grudnik P, Guzik K, Xieba BJ, Musielak B, Dömling A, Dubin G and Holak TA (2016) Structural basis for small molecule targeting of the programmed death ligand 1 (PD-L1). Oncotarget 7, 30323-30335.

32. Zarganes-Tzitzikas T, Konstantinidou M, Gao Y, Krzemien D, Zak K, Dubin G and Holak TA (2016) Inhibitors of programmed cell death 1 (PD-1): a patent review (2010-2015). Expert Opin Ther Pat 26, 973-977.

33. Zhan MM, Hu XQ, Liu XX, Ruan BF, Xu J and Liao C (2016) From monoclonal antibodies to small molecules: the development of inhibitors targeting the PD-1/PD-L1 pathway. Drug Discov Today 21, 1027-1036. 